\title{
SITUACIÓN DE LA VIOLENCIA DE GÉNERO EN COMUNIDADES INDÍGENAS Y MESTIZAS DE LOS MUNICIPIOS DE SIUNA Y ROSITA, RAAN
}

\author{
Jacoba del Carmen Dávila Molina ${ }^{[1]}$ \\ Lilia del Rosario Montoya Leal ${ }^{[2]}$ \\ María Cristina Feliciano ${ }^{[3]}$ \\ Ada Elieth Madrigal Ramírez ${ }^{[4]}$
}

\section{Resumen}

Esta investigación se realizó en cuatro comunidades mestizas y una indígena, en los municipios de Siuna y Rosita de la Región Autónoma del Atlántico Norte de Nicaragua en el 2010, la cual aborda la violencia basada en género. La metodología que se utilizó fue cualitativa y recoge las vivencias, percepciones y sentimientos de las mujeres víctimas de violencia y de las instancias encargadas de la atención a estos casos. Para el levantamiento de la información se utilizaron las técnicas de entrevista guiada y observación, las que se aplicaron a los grupos formados por representantes de las instituciones encargadas de hacer justicia, líderes comunitarios y mujeres víctimas de la violencia.

Los resultados muestran que existen mujeres en el área rural afectadas en sus derechos y deberes, que merecen una atención especial por parte de las instancias competentes, y que en su estado de vulnerabilidad no afrontan la situación de manera adecuada dado que este tipo de acontecimiento, que pone en riesgo su integridad física y moral, donde prevalece la violencia psicológica, seguida de la violencia física, económica y sexual, es aceptada hasta el punto de convertirse en su propia manera de vivir. En la investigación se identificaron dos rutas críticas a donde la minoría de mujeres entrevistadas ha acudido. Se cuenta con instituciones que brindan atención especializada a las mujeres; sin embargo, no se dispone del apoyo de la sociedad, hay carencia de recursos económicos y humanos para dar respuesta de manera beligerantes a esta problemática.

Palabras clave: víctima y victimario, ruta crítica, violencia de género, proceso judicial.

\footnotetext{
[1] Máster en Salud Pública. Coordinadora CEIMM, URACCAN Las Minas. adicarsiuna@yahoo.com.

[2] Licenciada en Ciencias Sociales. Docente tiempo completo URACCAN Las Minas. lilialeal14@yahoo.com.

[3] Licenciada en Administración, CEIMM URACCAN Las Minas. wakisa7@yahoo.com.mx

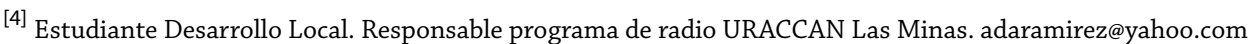




\section{Summary}

This research is related to violence based on gender, and it was conducted in four mestizo communities and one indigenous community, from the municipalities of Siuna and Rosita in the North Autonomous Atlantic Region of Nicaragua in the year 2010. The methodology used was qualitative and it reflects the experiences, perceptions and feelings of women victims of violence and the institutions responsible for the attention of these cases. The information was gather using guided interview techniques and observation, which were applied to the groups formed by representatives of institutions responsible to apply justice, community leaders and women victims of violence.

he results show that there are women in the rural areas who are affected in their rights and duties, whom deserves special attention by the competent authorities, and that due to their vulnerable state they do not face the situation appropriately, because this type of event even though it threatens their physical and moral integrity, and the psychological violence is prevalent, followed by physical, economic and sexual violence, it is accepted to the point of becoming their own way of living. The research identified two critical paths where the minority of women interviewed had attended. Although they are institutions that provide specialized care for women, however, there is no support from the society; there is lack of financial and human resources to respond in a belligerent way to this problem.

Keywords: victim and perpetrator, critical path, gender violence, prosecution.

\section{Introducción}

Esta investigación analiza la situación de la violencia que sufren las mujeres en las comunidades mestizas e indígenas de los municipios de Siuna y Rosita en la Región Autónoma Atlántico Norte de Nicaragua.

La perspectiva es crear el mapa de la ruta crítica de acceso a la justicia de las mujeres que son víctimas de violencia, e identificar situaciones, percepciones y acciones de las mujeres afectadas por la violencia basada en género, $y$, describir las percepciones, creencias y mitos de las personas que prestan servicios a nivel local, municipal y departamental en los casos de violencia.

Según la Organización Panamericana de la Salud (OPS), en América Latina, la violencia intrafamiliar afecta a una de cada tres mujeres, para ello se han realizado una serie de acuerdos internacionales para prevenir y erradicar la violencia contra la mujer en la última década, y como resultado de esos acuerdos, la mayoría de los países de la región han formulado legislaciones y políticas para enfrentar tal situación. Antes 
bien, no en todos ellos se han concretado reglamentaciones, instrumentos, recursos y políticas que hagan efectivas tales medidas (Marijke Velzeboer, OPS, p. 7).

La violencia en todas sus manifestaciones constituye una expresión más de la discriminación de género y debe ser considerada como una violencia de los derechos humanos. Es necesario analizar la magnitud, gravedad y dimensión del problema, ya que este hecho está muy extendido y supera el nivel de educación, situación social, situación económica, lugar de residencia, situación jurídica, etnia y edad.

Hoy en día se ha avanzado en la atención a las víctimas de violencia, a través de organización y áreas de atención a las mismas; empero, se carece de información que circule ampliamente en aquellos lugares de difícil acceso. A pesar de este avance aún existen ciertos grupos de poblaciones que viven en completo desconocimiento de esta situación, ya que muchas mujeres son abusadas, maltratadas y no toman ningún tipo de medidas, en este caso las mujeres de las áreas rurales e indígenas.

\section{Revisión de literatura}

\section{Ruta crítica de acceso a la justicia de mujeres víctimas de violencia basada en género}

Se percibe por ruta crítica el proceso que:

se construye a partir de decisiones tomadas y acciones ejecutadas por las mujeres afectadas por la violencia intrafamiliar y las respuestas encontradas en su búsqueda de soluciones. Este es un proceso reiterativo constituido tanto por los factores impulsores e inhibidores relacionados con las mujeres afectadas y las acciones emprendidas por éstas, como por la respuesta social encontrada, lo que a su vez se convierte en una parte determinante de la Ruta Crítica (OPS, 200o, p. 89).

Cabe destacar que una de las recomendaciones dadas por la investigación de la OPS (2000), se releva el rol activo ciudadano de las mujeres sobrevivientes en este proceso, y cómo los agentes prestatarios de servicios deben ser eficaces en su apoyo y acompañamiento. Es decir, entender que este "es un proceso transformador del que cada mujer es protagonista" (OPS, 2000, p. 108).

\section{Situaciones, percepciones y acciones de las mujeres afectadas por la violencia}

Los miembros de la familia viven situaciones y relaciones violentas como una forma natural de estar en el mundo, de relacionarse y resolver los conflictos y diferencias que surgen entre ellas y ellos. Las parejas despliegan frente a sus hijos e hijas modelos violentos de relación. Habría pues, una "política sexual" que va siendo aprobada a 
través de la socialización de niños y niñas y la internalización de las normas fundamentales del patriarcado que van afirmando la idea de la superioridad masculina en forma generalizada y que le garantiza una posición simbólica y social superior en la sociedad (Millet, 1970). Estos hijos e hijas aprenden estos patrones y posteriormente transmiten lo mismo a sus descendientes, perpetuando así una forma de convivencia que genera múltiples costos sociales, severos trastornos comunicacionales al interior de la familia e intensos sentimientos de inseguridad, frustración, resentimiento, miedo, impotencia y sufrimiento (Pateman, 1995 citado por Departamento de Estudios y Capacitación, la Unidad VIF del Servicio Nacional de la Mujer 2007, p. 10).

A pesar de la fuerza que tienen estos factores inhibidores internos y externos sobre la subjetividad de las mujeres en su decisión de comenzar una vida libre de violencia, existen situaciones límites objetivadas por éstas como aquellas que ponen en riesgo sus vidas y la de terceras personas de importancia para ellas. La OPS denomina estas situaciones como factores precipitantes: "si bien no existe un único factor precipitante o única razón por la que las mujeres inician una Ruta Crítica, generalmente el punto de saturación está asociado a momentos particulares de agresión." (OPS, 2000: 102).

\section{Percepciones, creencias y mitos de las personas que prestan servicios a nivel local, municipal y departamental en los casos de violencia}

Primero se define cultura, ya que los fenómenos que se abordarán en esta investigación remiten, necesariamente, a un determinado contexto de relaciones y producciones socioculturales. Freud, en el Malestar de la Cultura, entiende a la cultura como la "suma de las producciones e instituciones que distancian nuestra vida de la de nuestros antecesores animales y que sirven a dos fines: proteger al hombre contra la naturaleza y regular las relaciones de los hombres entre sí" (Freud, 1930: 3033). Si bien, se puede decir que todos los fenómenos sociales son productos culturales, cabe enfatizar que las dinámicas humanas están sobre determinadas por la ideología que instituye y promueve una determinada cultura; imperando, actualmente en nuestra sociedad, la cultura patriarcal (Parra M, 2002, p. 5).

A la vez, este concepto hace referencia a posiciones, relaciones y prácticas sociales que diferencian a los colectivos que conforman una determinada sociedad. En este sentido, la ideología da cuenta de las interacciones intergrupales y su relación con las desigualdades de poder entre los grupos sociales; es decir, ésta remite a la diferenciación social y, a la idea de oposición y dominación de los grupos. Por consiguiente, la cultura de ideología patriarcal se instaura con la división sexual del trabajo, donde el papel del hombre consiste en proporcionar la alimentación y los instrumentos de trabajo necesarios para ello, mientras que a las mujeres las posicionan dentro de las prácticas sociales que giran entorno a la maternidad y a las labores domésticas. Es decir: "esta ideología promueve una división sexual del trabajo, por la cual los hombres son asignados a la producción y al ámbito público mientras que las mujeres 
lo son a la reproducción y al ámbito privado y doméstico”, según Coria (1991, p. 17), citado por Parra M, 2002, p. 5).

Desde un análisis de género, la violencia hacia la mujer es un fenómeno cultural de profunda raigambre histórica, inserto en una forma de organización social y en un sistema de creencias culturales que determina prácticas, comportamientos y estilos de relación. Estas creencias subyacen y están en la base de la ideología patriarcal que se expresa y subordina a las mujeres al dominio y control masculino hegemónico (Departamento de Estudios y Capacitación, la Unidad VIF del Servicio Nacional de la Mujer. 2007, p. 8).

El género constituye una de las determinantes principales de las organizaciones sociales. La masculinidad y la femineidad son expectativas construidas socialmente y no categorías determinadas biológicamente. La pertenencia a un determinado género, al igual que la clase o la etnia, influye en las relaciones sociales de los seres humanos, delineando mayores o menores oportunidades, acceso a recursos y a estatus de privilegio, estando las mujeres permanentemente en una situación de inequidad respecto de los hombres (Rodríguez, 2001 citado por el Departamento de Estudios y Capacitación, la Unidad VIF del Servicio Nacional de la Mujer. 2007, p. 8)

Hoy existe consenso en señalar que no hay un factor que explique por sí solo las conductas violentas. En la actualidad, el modelo que mejor da cuenta de la complejidad de la violencia es el modelo ecológico, el cual incluye aspectos de marcos explicativos psicológicos, sistémico-familiares, económicos y socioculturales. Este enfoque modela la realidad en la interacción recíproca de cuatro niveles de sistemas: individual, microsistema, exosistema y macrosistema (Bronfenbrenner, 1987. Citado por Departamento de Estudios y Capacitación, la Unidad VIF del Servicio Nacional de la Mujer 2007, p. 12).

\section{Materiales y método}

Esta investigación se realizó en cuatro comunidades mestizas y una indígena, en los municipios de Siuna y Rosita de la Región Autónoma del Atlántico Norte de Nicaragua desde el enfoque del paradigma cualitativo, el cual señala que cuando reducimos las palabras y los actos de las personas a ecuaciones estadísticas, se pierde de vista el aspecto humano de la vida social. Si estudiamos a las personas cualitativamente llegamos a conocerlas en lo personal y a experimentar lo que ellas sienten en sus luchas cotidianas en la sociedad. (Taylor y Bogdan, 1996:21). Es ahí donde esta investigación recoge esas vivencias, percepciones, sentimientos de las mujeres víctimas de violencia de género . 
Es descriptiva, ya que permite comprender los resultados obtenidos, especificar las propiedades, las características y los perfiles de las personas y grupos que se sometan a un análisis.

Se utilizó la entrevista guiada y observación, aplicadas a los grupos conformados por representantes de las instituciones encargadas de hacer justicia, así como a las mujeres víctimas de violencia y líderes comunitarios. Se observó las conductas y las conversaciones en la participación, el retraimiento, la comunicación, los gestos y el silencio de las personas.

\section{Resultados y discusión}

\section{Ruta crítica de acceso a la justicia de mujeres víctimas de violencia basada en género}

Para darnos cuenta de la ruta crítica de acceso a la justicia de mujeres que han sido víctimas de la violencia, se recurrió a las instancia que de una u otra forma están vinculadas con la problemática, para ello se entrevistó a la defensora del movimiento de mujeres, representante de la PDDH, psicóloga, trabajadora social y jefa de la Comisaría de la Mujer, niñez y adolescencia, jueces, fiscales, médico forense; todos estos con incidencia a nivel departamental. A nivel comunal se entrevistó al Consejo de anciano, facilitador judicial, pastor y síndico.

En esta investigación se identificaron dos rutas:

\section{Primera ruta crítica}

En el caso de la mujer su primera instancia es el movimiento de mujeres "Paula Mendoza Vega", en el Municipio de Siuna, es atendida por la defensora quien registra el caso y valora el problema, si es leve y la víctima solicita mediación está facultada para resolverlo. La mediación consiste en que ambas partes se ponen de acuerdo (víctimavictimario) y firman conscientes que si no cumple una de las partes, en este caso el victimario, la víctima puede recurrir a otras instancia judiciales y seguir el proceso.

Si el caso amerita una atención especializada, la defensora refiere el caso a la Comisaría de la Mujer y le acompaña en todo el proceso. En esta instancia la víctima es valorada por la trabajadora social e interpone la denuncia ante la investigadora, quien determina si es necesario remitirla al médico forense, quien se encarga de realizar la valoración médica y emite el dictamen, donde determina si la víctima necesita una atención psicológica por las lesiones encontradas o la refiere nuevamente a la investigadora; reunidas todas las diligencias, es transferido el caso al Ministerio Público, quien clasifica los diferentes casos y los transfiere según su competencia en dos vías: al juzgado local o Juzgado de distrito, según el caso. 


\section{GÉNERO E IDENTIDAD}

Además, en el acceso de las víctimas a la justicia se hace a través del sistema de referencia y contra referencia al que las mujeres víctimas de violencia acuden. Las instancias que realizan este canal son principalmente las organizaciones de mujeres, que es el lugar donde llega de primero la víctima y rompe el silencio. Se presentan muchos casos en donde la víctima solicita que se resuelva el problema de violencia a través de la mediación, aunque las instancias donde acuden, les recomiendan a las mujeres que nos es conveniente realizar mediaciones. Según lo establece en el nuevo código procesal penal. Por lo tanto se remiten a la Comisaría de la Mujer y se acompaña en todo el proceso judicial.

\section{Segunda ruta crítica}

En este caso se destacan las comunidades estudiadas que corresponden al municipio de Rosita, las cuales carecen de instancias judiciales en atención a las víctimas de violencia intrafamiliar y por lo tanto su proceso de atención inicia en la comunidad ante el facilitador judicial, quien según el caso realiza la mediación o lo transfiere al Ministerio de Salud, donde se realiza la valoración médica y posteriormente este lo remite al movimiento de mujeres "Nora Astorga", quienes les acompañan a la policía del municipio, y luego este se encarga de enviar el caso a la Comisaría de la Mujer en Siuna o fiscalía, donde se continua el proceso como se observó en la ruta anterior.

El tiempo que dura este proceso cuando ya se inicia la ruta de atención es diversa, cuando el victimario está detenido se tiene un período como máximo de 48 horas para contar con todas las diligencias y ser remitido, en el caso de la víctima, a la fiscalía y el detenido a la orden del juez. Por otra parte si no está detenido se cuenta con un período de 20 días como máximo para continuar las investigaciones y reunir todas las diligencias y así ser remitido el caso al Ministerio Público, de no ser así al cumplir con el período, se cierra el caso administrativamente y se abandona el proceso.

A nivel comunal, las mujeres para resolver el problema de violencia llevan a cabo procedimientos propios, que tiene que ver con las características y formas de organización de la comunidad. En el caso particular de Wasakin, comunidad indígena tuahka, donde las mujeres víctimas de violencia en su mayoría solicitan ayuda a figuras jurídicas indígena de derechos consuetudinarios en las comunidades como son: reverendo, consejo de anciano, wihta, en muchos casos se les impone castigos a los agresores y se hacen arreglos de parejas, también realizan charlas y consejerías con la pareja afectada, cuando a pesar de la intervención de esta representación sigue el problema, se recomienda a la víctima ir a la policía a interponer la denuncia porque ya no está en las manos de la comunidad, pero gran parte de los casos no se denuncian y así convive la pareja. 
En el caso de las comunidades mestizas se desarrollan diferentes procesos en que las mujeres víctimas recorren para resolver el problema de violencia, se resalta que las mujeres le cuentan su problema a una persona conocida y esta le apoya a ir a donde el facilitador judicial, mediador, a la policía, o al movimiento de mujeres, esto depende de la gravedad del problema y de la confianza que tengan las mujeres víctimas ante las instancias.

Por otro lado, también las mujeres víctimas de violencia pueden iniciar este proceso en el Centro de Salud donde recurren por algún problema de salud producto de la violencia que sufren, puede ser que la paciente presente heridas, golpes, dolor, al detectar el problema de violencia es competencia de esta institución remitir el caso a la Comisaría de la Mujer; sin embargo, existen limitantes para realizar un diagnóstico en el que se determine si el problema de salud fue procedente de violencia.

\section{Situaciones, percepciones y acciones de las mujeres afectadas por la violencia basada en género}

La violencia se percibe como los desacuerdos que surgen en las parejas o familia, que provocan afectaciones en las personas y en la familia, ya sean psicológicas, físicas o económicas, es vivir una mala vida, es la violación a los derechos de las personas, derecho a la educación, a la salud, a la vida. Se destaca como violencia el abandono de hijos e hijas por parte de los padres en donde se le niegan los derechos humanos fundamentales establecidos en las legislaciones internacionales.

La violencia es reconocida como algo que se ha aprendido por las conductas observadas principalmente en el hogar y en los lugares de concurrencia, pero además se percibe que la violencia debe ser pública desde el solo hecho que hay una violación a los derechos humanos, y es ahí donde deja de ser algo privado, ya que es un problema social y de salud pública, en donde hay una afectación ya sea física, psicológica o emocional, que requiere de una responsabilidad social y legal que garantice una vida sin violencia y una seguridad a la víctima.

Para las mujeres indígenas se señala como factor que prevalece y se justifica la violencia el factor cultural, reflejado este en un mandato religioso donde a las mujeres se les ha enseñado a aguantar, ser sumisas, tímidas y calladas, haciendo énfasis en que el matrimonio es para toda la vida y por eso se ven fuertemente influidas y consideran que deben de aguantar. Otro de los factores fue el económico, porque refieren que los hombres son los proveedores y si lo denuncian no tendrán ese ingreso, existe una dependencia económica.

Muchas veces las mujeres permiten que el hombre las mande y tome el poder sobre ellas; empero, cuando se unen en matrimonio es para formar una familia y vivir en paz, pero la creencia que prevalece en ellos, hace que este tome el control sobre las 
mismas violentándole sus derechos como seres humanos, prevalece la percepción que los hombre son los primeros y siempre serán primeros y eso es lo fundamentan en la creación de los seres humanos haciendo énfasis en Adán y Eva. En la mayoría de los casos, las mujeres son muy humildes y tienen mucha paciencia, y refieren aguantar por sus hijos e hijas. Por otra parte, existen mujeres que han pasado por otros procesos de sensibilización y buscan los mecanismos para que sus derechos sean respetados.

En los casos estudiados de las comunidades mestizas, coincide con las indígenas y se resalta el patriarcado como una de las bases principales en donde se fundamenta el dominio y control de los hombres hacia las mujeres y eso da paso a las agresiones ya sean verbales o físicas. Otro de los aspectos que sobresale son los celos como un elemento clave para maltratar a las mujeres y por último, pero con un gran significado se destaca la falta de conocimiento de las mujeres sobre sus derechos.

Un $98 \%$ de las mujeres involucrada en la investigación han vivido o viven actualmente la violencia, en sus diferentes tipologías. Se encontró que en la mayoría de los casos las victimas sufren violencia psicológica, física, económica o patrimonial y sexual. Dentro de la violencia psicológica las mujeres refieren agresiones verbales, humillaciones, temor, ignorancia, aislamiento y amenazas de muerte con diferentes objetos (machete, arma de fuego, leño, entre otros).

Además, las mismas tienen miedo y se desaniman por una respuesta caracterizada por la falta de efectividad y atención adecuada desde las instancias correspondientes del estado (Policía, Fiscalía y juzgados). Se percibe que las víctimas no reciben respuesta adecuada de las autoridades. Algunas creen que si van a la policía de su localidad podrían gastar dinero y no tener una respuesta adecuada a su problema.

Algunas mujeres víctimas han logrado romper el silencio y han denunciado su caso, otras en su mayoría piensan que al buscar ayuda, no lograrán nada y creen que más bien pueden encontrar la muerte ya que han sido amenazadas por sus cónyugue y perciben poca protección policial, y por lo tanto sienten que el agresor al darse cuenta que lo ha denunciado, a su regreso la matará o la situación se agravará aún mas.

En el caso específico de las mujeres indígenas, una de las situaciones por las cuales no denuncian la violencia es por la barrera lingüística, además de todo lo que se ha referido anteriormente. Consideran que la denuncia de casos de violencia se ha incrementado, porque hay más conciencia de parte de las mujeres sobre sus derechos, éstas rechazan más constantemente el control, los celos y los maltratos psicológicos y físicos. No obstante, piensan que es importante la sensibilización hacia todos los grupos sociales y fundamentalmente hacia los hombres y a las instancias que hacen justicia. 


\section{Percepciones, creencias y mitos de las personas que prestan servicios a nivel} local, municipal y departamental en los casos de violencia

Los resultados encontrados en el sistema de justicia refleja que la violencia hacia la mujer es producto de la mala información que existe en el tema de violencia, tiene que ver con la cultura ya que se ha ido inculcando que las mujeres son como objeto y no se le da el valor que merece como ser humano. También perciben que al ingerir alcohol o el mismo consumo de droga, el desempleo agrava aún más el problema de la violencia. Además se da más porque las mujeres que son violentadas no denuncian a sus agresores, es necesario romper ese silencio y buscar ayuda psicológica y jurídica para que ellas tengan respuesta por parte de las autoridades competentes.

A pesar de las luchas de las mujeres por sus derechos humanos, hay resistencia del sexo masculino por entender esos derechos y respetarlos, es importante que toda la sociedad se sienta comprometida para reducir la violencia.

Se reconoce que la educación recibida en las familias, escuela e iglesias, influye mucho en la desigualdad de oportunidades y derechos entre hombres y mujeres y que han transcurrido muchos años de estas prácticas y se sigue trasmitiendo en nuestra generación.

Otras de las situaciones por las cuales se considera que las mujeres sufren violencia es por la dependencia económica y emocional, por eso cuando ya está en el ciclo de la violencia es muy difícil que ella logre salir, ya que esta dependencia no le permite visibilizar otra opción de vida, para ella y sus hijos e hijas, por ende aguantan muchos años y en reiteradas ocasiones hasta la muerte.

Como resultado de las campañas de sensibilización de lucha por una sociedad sin violencia impulsadas por las organizaciones que trabajan la temática, así como los programas radiales u otro tipo de información hacia la sociedad en general; esto ha permitido que la mujer tome la decisión de denunciar, por esa razón se ve que el universo de las mujeres maltratadas es mayor; porque los hombres si bien es cierto que también son objeto de agresiones físicas y de lesiones psíquicas, además es cierto que es un grupo menor desde el punto de vista estadístico, menor por el hecho de que así fueron formados.

Se percibe en las mujeres víctimas de violencia cuando llegan a denunciar su timidez, crisis nerviosas y depresivas, razón que sienten porque no hay seguridad jurídica para ellas, piensan de que las autoridades no van a hacer lo necesario, hay desconfianza de las mujeres y temor por lo que les pueda pasar después de haber denunciado y en todo el proceso, por eso algunas abandonan el caso, de igual manera piensan de que no les van a creer lo que ellas digan. Tienen temor que el caso quede impugne y que se vaya agudizar más y la víctima está en riesgo de perder su vida. Se 
han presentado casos de violencia intrafamiliar, sobre todo, los casos realizados por jurados de consciencia, que han dejado en libertad a los victimarios, aún teniendo visibles todas las diligencias.

En la ruta crítica que se les da a los casos se les re victimiza a las mujeres, de una manera inconsciente, o sea no se quiere re victimizar, pero al no contar con todos los recursos, no se tiene al personal adecuado, capacitación necesaria para atender un caso de violencia, ese es un problema serio en el sistema, tiene que ver desde que la mujer es objeto de violencia y pone la denuncia, después va al médico forense, la fiscalía, ir al juzgado que constituye una angustia. La mujer tiene que ir contando lo que vivió a cada funcionario que la atiende, y no todos están capacitados o sensibles para atenderla.

La violencia en todas sus expresiones genera un impacto en la salud en la víctima, y este impacto será más negativo en la medida de que esta sea menor de edad, precisamente por su inmadurez fisiológica y psicológica, se hace más difícil que se supere las secuelas, los estigmas de que ha sido objeto de violencia una niña, esto porque en una mujer adulta hay capacidad de razonamiento, hay capacidad de discernir; en una menor esas capacidades no están desarrolladas, por lo tanto las secuelas marcan de por vida a las menores.

Hay una gran preocupación de parte del sistema de Justicia por los casos que se realizan con jurados de consciencia, en los cuales se manifiesta que la sociedad no es responsable de sus decisiones en los juicios, hay insatisfacción del personal judicial, como también de las mujeres víctimas y sus familiares. Pero, con la entrada en vigencia del nuevo código penal los jurados no tienen que ver en los delitos de carácter sexual y en los casos de violencia intrafamiliar; entonces, se proyecta una visión positiva para que la gente denuncie y se fomente la justicia, en el caso de los jueces es difícil que si se presentan todas la prueba, va a absolver a un violador o a una persona acusada de violencia intrafamiliar

La respuesta de todo el sistema social ante la violencia intrafamiliar que ofrecen en el ámbito local las instituciones gubernamentales y no gubernamentales. Tanto el Estado, los organismos, la sociedad civil, en coordinación y unidos, incluso la misma víctima tiene que colaborar, así lograremos reducir o disminuir el alto índice de la violencia contra la mujer.

No se ha hecho lo mejor para resolver los problemas de violencia, pero si se realizan a diario, los esfuerzos para incidir en que este fenómeno disminuya. Sería iluso creer que este fenómeno se va a erradicar, se dejaría de existir como sociedad si se creyera que a un plazo determinado se va a erradicar, precisamente cuando los valores negativos están arraigados en la conducta humana, la violencia va seguir existiendo y el trabajo de estas organizaciones y de las instituciones es y seguirá siendo valioso. 


\section{Conclusiones}

Existen mujeres en el área rural que requieren atención especial por parte de las instancias competentes, ya que en su estado de vulnerabilidad no afrontan la situación de manera adecuada y esto pone en riesgo su integridad física y moral hasta el punto de convertirlas en su propia manera de vivir.

El $98 \%$ de las mujeres entrevistadas vive o ha vivido violencia durante toda su vida. Se reconoce que la violencia es un delito, pero muchas de ellas por temor e inseguridad siguen soportando en silencio esta violación a sus derechos.

Se identificaron dos rutas críticas en las que la minoría de mujeres entrevistadas han acudido a instituciones que brindan atención especializada; empero, no se cuenta con el apoyo de la sociedad, hay carencia de recursos económicos y humanos para dar respuesta de manera beligerante a esta problemática.

Con la vigencia del nuevo código penal se ha avanzado en el sistema de justicia, ya que cuando se resolvían los casos de violaciones con jurados de consciencia, en su mayoría a los agresores se les declaraba inocentes, aunque se contara con todas las diligencias, por lo que actualmente la justicia a las víctimas de violencia es más eficiente.

\section{Lista de referencias}

Departamento de Estudios y Capacitación (2009).. Análisis y Evaluación de la Ruta Crítica en Mujeres Afectadas por Violencia en la Relación de pareja. Documento de Trabajo $\mathrm{N}^{\circ} 107$.

GARCIA, Yomaira (2006), Caracterización de la violencia hacia la mujer en el departamento del Atlántico: Un estudio preliminar, Universidad Simón BolívarProyecto cero Violencia hacia las mujeres, UNIFEM.

Herrera Teresa (2004). Estudio de prevalencia de la violencia doméstica en Uruguay",Banco Inticano de Desarrollo, Montevideo.

Marijke Velzeboer (200o). Programa Mujer Salud y Desarrollo. Organización panamericana de Salud.

Velásquez, S. (2005). Violencia cotidiana, violencia de género. Buenos Aires: Editorial, Paidos.

OPS/OMS PROGRAMA, MUJER, SALUD Y DESARROLLO (2000). Ruta crítica por las mujeres afectadas de la violencia intrafamiliar en América Latina. (estudio de caso de diez países). 